Original Research

\title{
The Effect of Chinese Medicine for Rehabilitation of Discharged COVID- 19 Patients: A Protocol for Multi-Center Observational Study
}

Linda Li-Dan Zhong ${ }^{1,}{ }^{*}$, Yi-Ping Wong ${ }^{1}$, Bo Peng ${ }^{1}$, Zhi-Xiu Lin ${ }^{2}$, Vivian Chi-Woon Wong Taam ${ }^{3}$, Yi Luo ${ }^{3}$, Hai-Yong Chen ${ }^{3}$, Chao-Dong Chao ${ }^{4}$, Chor-Fung Wong ${ }^{5}$, Freddie Shung-Chi Tam ${ }^{6}$, Kui Chan ${ }^{7}$, KwanYiu Lee ${ }^{8}$, Lai-Fun $\mathrm{Ho}^{9}$, Alan Yat-Lun Wong ${ }^{10}$, Chi-Fung Choy ${ }^{11}$, Bacon Fung-Leung $\mathrm{Ng}{ }^{12}$, Rowena How-Wan Wong ${ }^{12}$, Yi-Bin Feng ${ }^{3}$, Ching Liong ${ }^{2}$, Zhao-Xiang Bian ${ }^{1,}{ }^{*}$, COVID-19 CM Research Working Group

1. School of Chinese Medicine, Hong Kong Baptist University, Hong Kong; E-Mails: Idzhong@hkbu.edu.hk; bzxiang@hkbu.edu.hk; ypwong@hkbu.edu.hk; scmcld@hkbu.edu.hk

2. School of Chinese Medicine, The Chinese University of Hong Kong; E-Mails: linzx@cuhk.edu.hk; liongching@cuhk.edu.hk

3. School of Chinese Medicine, LKS Faculty of Medicine, The University of Hong Kong; E-Mails: vcwwong@hku.hk; yiluo7@hku.hk; haiyong@hku.hk; yfeng@hku.hk

4. United Christian Nethersole Community Health Service - The Chinese University of Hong Kong Chinese Medicine Clinic cum Training and Research Centre (Tai Po District); E-Mail: cd.chao@ucn.org.hk

5. HKFTU Workers' Medical Clinics - Hong Kong Baptist University Chinese Medicine Clinic cum Training and Research Centre (North District); E-Mail: tcmchorfung@hotmail.com

6. Pok Oi Hospital - Hong Kong Baptist University Chinese Medicine Clinic cum Training and Research Centre (Kowloon City District); E-Mail: cmphmt1@pokoi.org.hk

7. The Hong Kong Tuberculosis Association - The University of Hong Kong Chinese Medicine Clinic cum Training and Research Centre (Southern District); E-Mail: michael.chan.tcm@gmail.com

8. Pok Oi Hospital - The Chinese University of Hong Kong Chinese Medicine Clinic cum Training and Research Centre (Yuen Long District); E-Mail: leekwanyiu1986@yahoo.com.hk

9. Pok Oi Hospital - The Chinese University of Hong Kong Chinese Medicine Clinic cum Training and Research Centre (Shatin District); E-Mail: midcoho@gmail.com

10. Haven of Hope - The Chinese University of Hong Kong Chinese Medicine Clinic cum Training and Research Centre (Sai Kung District); E-Mail: alanylwong@hohcs.org.hk

11. Department of Medicine, Tseung Kwan O Hospital, Hospital Authority, Hong Kong; E-Mail: ccf011@ha.org.hk

12. Chinese Medicine Department, Hospital Authority, Hong Kong; E-Mails: ngfl@ha.org.hk; rowena.wong@ha.org.hk

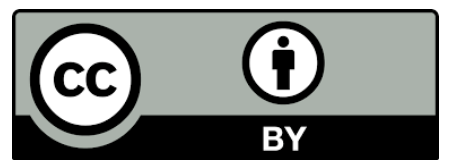


*Correspondence: Linda Li-Dan Zhong and Zhao-Xiang Bian; E-Mails: Idzhong@hkbu.edu.hk; bzxiang@hkbu.edu.hk

Academic Editors: Soo Liang Ooi and Dr Pak Sokcheon

Special Issue: Complementary, Traditional, and Integrative Medicine for COVID-19

OBM Integrative and Complementary Medicine

2021, volume 6 , issue 3

doi:10.21926/obm.icm.2103022
Received: May 24, 2021

Accepted: July 01, 2021

Published: July 13, 2021

\begin{abstract}
The COVID-19 pandemic has lasted for more than 16 months, and there have been over 169 million confirmed cases worldwide. Besides, after treatment with Western medicine or undergoing home quarantine, COVID-19 patients are still severely or mildly functionally impaired. Though COVID-19 patients were discharged from the hospital, most of them still exhibit certain clinical symptoms such as fatigue, poor appetite, shortness of breath, and poor sleep. The syndromes, linked with the Chinese Medicine (CM) body constitutions, could be due to pre-COVID-19 infections, suffering from the infection, or a post-infection consequence. $\mathrm{CM}$ has been used by humans for thousands of years in Asia, especially in Hong Kong, and it is gaining increasing attention and popularity. This study aimed to evaluate the efficacy of CM on alleviating the clinical symptoms of the discharged COVID-19 Patients. This was a multicenter observational and comparative study. One hundred and fifty participants discharged from Hong Kong hospitals were recruited. The patients received three to six months of treatment using $\mathrm{CM}$ and were assessed by questionnaires and lung function tests each month during the treatment period and on the $9^{\text {th }}$ month follow-up visit. In light of this global pandemic, we hope this study will bring new opportunities for $\mathrm{CM}$, and facilitate patient recovery and rehabilitation. We believe that this may be the key to promoting rehabilitation.
\end{abstract}

\title{
Keywords
}

COVID-19; rehabilitation; observational study; Chinese medicine; respiratory system; lung function; Quality of Life; body constitutions

\section{Introduction}

In March 2020, the World Health Organization (WHO) declared the outbreak of a coronavirus disease 2019 (COVID-19) to be a pandemic. Worldwide, governments are fighting the virus and, at the same time, taking action to contain its spread. Fortunately, the vaccines were ready and publicly available in early January 2021. In fact, COVID-19 has lasted for more than 16 months, with over 169 million confirmed cases all over the world [1]. Though after Western medicine treatment or undergoing home quarantine, the functionality of COVID-19 patients is still severely or mildly impaired. These include not only physical weakness but also problems of the pulmonary, physical, and psychosocial domains [2]. In 2003, Hong Kong experienced the outbreak of a severe acute 
respiratory syndrome (SARS) epidemic. A review of the publications during and after the SARS crisis enabled us to get an objective view of the true value of the adjuvant therapy using Chinese medicines (CMs) [3].

According to the WHO, most patients had mild or uncomplicated forms of COVID-19, while approximately $14 \%$ were estimated to be associated with a severe acute respiratory infection and required hospitalization and oxygen support, and $5 \%$ required admission to the intensive care unit (ICU) [4]. Even while patients with COVID-19 are discharged from hospitals, they still present significant clinical symptoms such as fatigue, poor appetite, shortness of breath, and poor sleep. They experience not only physical weakness but may also suffer from pulmonary, physical, and psychosocial problems. This disease has not been understood completely. Hence, its sequelae and long-term effects on pulmonary rehabilitation in COVID-19 need further evaluation.

For thousands of years, CM has been used by humans in Asia, and it is gaining increasing attention globally. Being the epicenter of the outbreak, China developed the National COVID-19 Diagnosis and Treatment Guidelines, and has constantly been updating information about the disease. To facilitate the implementation of integrative Chinese-Western Medicine in COVID-19 management, CM has been recommended in the 7th edition of the national guidelines released in March 2020 [5]. For this reason, $\mathrm{CM}$ rehabilitation treatment guidelines for those patients were also released simultaneously [6-10]. With the aim to strengthen the rehabilitation for health management of discharged COVID19 patients, and help them recover and return to society safely, CM has been used as a treatment in China during the outbreak. Some of the early papers reported that $>85 \%$ of COVID-19 infected patients in China were receiving CM treatment, with an overall effective rate of $\geq 90 \%$ [11]. Among them, the symptoms of majority of patients $(\geq 60 \%)$ improved markedly, while the illness of others (30\%) was stabilized [12].

Although the number of published papers on COVID-19 has increased, many questions still remain, and available treatment options are limited [5]. CM Practitioners (CMPs) in Hong Kong have extensive experience in using $\mathrm{CM}$ to prevent and treat disease. It is, therefore, important to draw on their experience and summarize the evidence for the effectiveness of CM on post-COVID-19 rehabilitation. Since early January 2020, there has been a response to the COVID-19 pandemic in Hong Kong. As of 20 May 2021, a total of 11,828 COVID-19 cases have been confirmed, and 11542 patients were discharged, according to the Centre for Health Protection of the Department of Health in Hong Kong [13].

\section{Materials and Methods}

\subsection{Overview of Study Design}

This was a multicenter observational study. One hundred and fifty discharged COVID-19 patients who are Hong Kong Chinese aged 18 years or older were recruited. The patients received three to six months of individualized CM treatment based on CM guidelines on COVID-19 rehabilitation, and individual clinical symptoms. All participants were assessed by questionnaires and lung function tests each month during the treatment period and on follow-up visit.

This study was divided into two parts:

Retrospective syndrome survey: Medical history of participants during COVID-19 hospitalization was collected during the baseline visit, after obtaining informed consent from the participants. This 
was done with the aim to delineate disease severity with the CM syndrome, which includes COVID19 diagnosis date, hospitalization period, and other chronic illnesses in the case report by semistructured questionnaire and the self-developed Body Constitutions Questionnaire.

CM therapeutic assessment: Participants were treated with CM based on syndrome differentiation. The improvement of clinical symptoms and the status of body constitutions were periodically evaluated. The examinations included clinical CM diagnostic pattern and clinical characteristics assessments, lung function tests, and quality of life assessment at each visit for nine months.

\subsection{Recruitment}

Participants who were discharged from Hong Kong hospitals and seek CM treatment at the Chinese Medicine Clinic cum Training and Research Centres (CMCTRs), which are the governmentsubsidized tri-partite CM out-patient clinics, were recruited. One hundred and fifty participants were found to be eligible for the program. We had seven clinical sites in total, and we assumed that 20-30 participants could be recruited from each site. The study protocol was approved by the corresponding Ethics Committee(s). Participants who had recovered from COVID-19 were enrolled upon their capacity to give written informed consent voluntarily.

\subsection{Eligibility Criteria}

Inclusion Criteria: We recruited participants (aged 18 years or older) under the COVID-19 Rehabilitation Program who had been previously diagnosed to be infected with COVID-19 and discharged from local hospitals after treatment with Western medicine, and with negative results from COVID-19 virus detection test.

Exclusion Criteria: Participants were excluded if they had one or more of the following: 1) inability to communicate (e.g., cognitive impairment), and 2) history of $\mathrm{CM}$ allergies.

\subsection{Interventions}

The participants received three to six months of CM treatment based on their recommended prescription, individual CM syndrome, and clinical symptoms [14-17]. Each participant was assessed monthly during the treatment and follow-up. The treatment and assessments were conducted by a registered $\mathrm{CM}$ practitioner with at least three years of clinical experience.

Treatment was offered based on the CM clinical practice guidelines for COVID-19 patients (The National Health Commission of the People's Republic of China, 2020), which has been suggested by numerous clinical studies and guidelines [14].

\subsection{Outcomes Measures}

The primary outcome includes the scores of CM Diagnostic Pattern \& Clinical Characteristics Assessments and Body Constitution Questionnaires using a Scoring Checklist, which covered two main syndromes (lung and spleen qi deficiency and qi and yin deficiency), and each included three major symptoms and five accompanying symptoms. A four-point scale $(0,2,4,6$ and $0,1,2,3)$ was used depending on symptom severity. The scores summed up to a range of 0 to 33, with higher scores indicating more severe levels of disease (Please refer to Appendix 1). Questionnaire on body 
constitution was classified into nine specific types of questionnaires based on Traditional Chinese Medicine (TCM) theories, which has been recognized by the China Association of Chinese Medicine as the standard of body constitution (China Association of Chinese Medicine, 2009) [18-20].

The secondary outcomes include forced expiratory volume in $1 \mathrm{~s}$ (FEV-1), lung function-related assessments (Lung Function Questionnaire, and the 6-min walk test), lung function test assessed by the handheld basic spirometry, quality of life (WHO-QOL BREF HK) and the frequency of clinic or hospital visits for Western medicine during the treatment and follow up period.

The key spirometry measurements include the Forced Vital Capacity (FVC) and Forced expiratory volume (FEV). FVC is the largest volume of air that the participants can forcefully exhale after breathing in as deeply as they can. A lower than normal FVC reading indicates restricted breathing. FEV-1 is the volume of air the participants can force out of their lungs in one second. Lower FEV-1 readings indicate more significant pulmonary obstruction.

Lung Function Questionnaire is a simple, brief, self-administered instrument to identify patients' suitability for further lung function evaluation. It is a five-items questionnaire about the breathing and/or cough ailments of the participants. It was validated in China and widely used in primary care [21].

The WHO Quality of Life Brief Assessment [WHOQOL-BREF (HK)] (Leung, K F; Tay, M., Cheng, S. S. W., Lin, 1997) was validated in Hong Kong and has been widely used in academia and by clinicians since 1997. This is a 5-point Likert scale with a total of 28 questions to identify the perceived QOL of the participants. There are four domains, namely physical health, psychological, social relationships, and environment domains with a maximum score of 100 .

\subsection{Participant Timeline}

The participants received three to six months of individualized CM treatment, based on CM guidelines on COVID-19 rehabilitation and individual clinical symptoms. Each participant was assessed by questionnaires and lung function tests monthly during the treatment period and on follow-up visit. (Please refer to Table 1)

Table 1 The Schedule for Outcome Measurement.

\begin{tabular}{|c|c|c|c|c|c|c|c|c|}
\hline \multirow{2}{*}{$\begin{array}{l}\text { Assessment } \\
\text { Visit }\end{array}$} & \multirow{2}{*}{$\begin{array}{l}\text { Baseline } \\
1\end{array}$} & \multicolumn{6}{|c|}{ Program Intervention } & \multirow{2}{*}{$\begin{array}{l}\text { Follow-up } \\
8\end{array}$} \\
\hline & & 2 & 3 & 4 & $5^{*}$ & 6* & 7* & \\
\hline Study Months & 0 & 1 & 2 & 3 & $4^{*}$ & $5^{*}$ & $6^{*}$ & 9 \\
\hline Informed Consent & $\mathrm{V}$ & & & & & & & \\
\hline History & $\mathrm{V}$ & & & & & & & \\
\hline $\begin{array}{l}\text { CM Diagnostic Pattern \& Clinical Characteristics } \\
\text { Assessments }\end{array}$ & $\mathrm{V}$ & $\mathrm{v}$ & $\mathrm{V}$ & $\mathrm{v}$ & $\mathrm{V}$ & $\mathrm{V}$ & $\mathrm{V}$ & $\mathrm{V}$ \\
\hline Lung Function Assessments FEV/FVC 6-MWT & $\mathrm{V}$ & $\sqrt{ }$ & $\mathrm{V}$ & v & $\mathrm{V}$ & $\mathrm{V}$ & $\mathrm{V}$ & $\mathrm{V}$ \\
\hline $\begin{array}{l}\text { Quality of Life Assessments (WHO-QOL BREF } \\
\text { HK) }\end{array}$ & $\mathrm{v}$ & 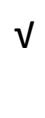 & $\mathrm{v}$ & $\mathrm{v}$ & $\mathrm{v}$ & $\mathrm{v}$ & $\mathrm{V}$ & $\mathrm{v}$ \\
\hline Body Constitution Questionnaires (CCMQ) & $\mathrm{V}$ & $\sqrt{ }$ & $\mathrm{V}$ & 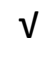 & $\mathrm{V}$ & $\mathrm{V}$ & $\checkmark$ & $\mathrm{V}$ \\
\hline
\end{tabular}




\begin{tabular}{llllllllllll}
\hline Assessments on the Frequency of WM Visits & $v$ & $v$ & $v$ & $v$ & $v$ & $v$ & $v$ & $v$ & \\
\hline
\end{tabular}

* The extended treatment is optional.

Remarks: There will be 8 study visits in total

Visit 1: is a Baseline visit

Visit 2 to Visit 7: Patients will receive Chinese medicine treatment and will be assessed by questionnaires and lung function tests on the same day

Visit 8: follow-up visit after three months at V7

\subsection{Data Collection, Management, and Analysis}

All analyses were conducted according to the intention-to-treat (ITT) principle. Missing values were inputted by the last-observation-carried-forward method. The statistical analysis was performed using the Statistical Product and Service Solutions (SPSS) for Windows version 25.0. A Pvalue of $<0.05$ was considered statistically significant. Numerical variables needed to be first tested for normality and were then reported as mean if the data were satisfied with normal distribution; otherwise, the median was used. Comparisons of numerical variables before and after Program intervention were analyzed by repeated measures of ANOVA for categorical variables, and chisquared test or Fisher's exact test were used for analysis.

\subsection{Ethics Statement}

This study protocol was approved by Hong Kong Baptist University Research Ethics Committee (Approval no. REC/19-20/0468) and Hospital Authority (Approval no. HA 105/48 PT35), and was registered at the ClinicalTrials.gov on 3 September 2020 (NCT04544605).

\section{Discussion}

The role of $\mathrm{CM}$ is to enhance anti-epidemic efforts, particularly in relation to prevention and rehabilitation treatment, and to raise the capability of CMPs in the areas. To date, there is no reported long-term treatment and no long-term observational study for the discharged COVID-19 patients. Our team has gained preliminary satisfactory treatment outcomes with the application of $\mathrm{CM}$ for patients who still exhibit certain clinical symptoms such as fatigue, poor appetite, shortness of breath, and poor sleep.

Today the Chinese alternative medicine branch, collectively known as $\mathrm{CM}$, is becoming increasingly relevant as the world fights the deadly spread of the coronavirus disease. CMs have their own characteristics such as holistic concept, the balance of Yin and Yang, syndrome differentiation and treatment, and strengthening the body resistance to eliminate pathogenic factors.

This study will provide evidence for further research on the changes in body constitutions, and evaluate the therapeutic effects of $\mathrm{CM}$ based on individualized treatment. Improvement in clinical symptoms and body constitution could also be assessed specifically in the Hong Kong population. The effects of CM on the respiratory system by lung function assessments included FEV in $1 \mathrm{~s}$, a 6min walk test, and the Lung Function Questionnaire. In light of the global pandemic, the development of $\mathrm{CM}$ may bring new opportunities and hope to provide references for control and 
rehabilitation of COVID-19 patients around the world, and we believe that this may be the key to promoting rehabilitation resolution.

\section{Acknowledgments}

This research was financially supported by Hong Kong Hospital Authority Research Project (Ref Code: HA 105/48 PT35). The authors would also like to thank all the medical staff who dedicated their time and efforts in managing patients during the Covid-19 pandemic.

\section{Additional Materials}

The following additional materials are uploaded at the page of this paper.

1. Appendix 1: The scores of CM Diagnostic Pattern \& Clinical Characteristics Assessments.

\section{Author Contributions}

All authors contributed equally to this study and submission. The author(s) read and approved the final manuscript.

\section{Funding}

This research was financially supported by Hong Kong Hospital Authority Research Project: Special Chinese Medicine Out-patient Programme for Discharged Covid-19 Patients - Observational Study (Ref Code: HA 105/48 PT35).

\section{Competing Interests}

The authors have declared that no competing interests exist.

\section{References}

1. World Health Organization (WHO). WHO coronavirus (COVID-19) dashboard [Internet]. Available from: https://covid19.who.int/.

2. Bij de Vaate E, Gerrits KH, Goossens PH. Personalized recovery of severe COVID19: Rehabilitation from the perspective of patient needs. Eur J Clin Invest. 2020; 50: e13325.

3. Leung PC. The efficacy of Chinese medicine for SARS: A review of Chinese publications after the crisis. Am J Chinese Med. 2007; 35: 575-581.

4. World Health Organization. Clinical management of severe acute respiratory infection (SARI) when COVID-19 disease is suspected [Internet]. Interim Guidance; 2020. Available from: https://apps.who.int/iris/handle/10665/331446.

5. Ho LT, Chan KK, Chung VC, Leung TH. Highlights of traditional Chinese medicine frontline expert advice in the China national guideline for COVID-19. Eur J Integr Med. 2020; 36: 101116.

6. Diagnosis and treatment protocol for novel coronavirus pneumonia. National Health Commission \& State Administration of Traditional Chinese Medicine; 2020. Available from: https://www.chinadaily.com.cn/pdf/2020/1.Clinical.Protocols.for.the.Diagnosis.and.Treatmen t.of.COVID-19.V7.pdf. 
7. Chinese Association of Rehabilitation Medicine, Respiratory Rehabilitation Committee of Chinese Association of Rehabilitation Medicine, Cardiopulmonary Rehabilitation Group of Chinese Society of Physical Medicine and Rehabilitation. [Recommendations for respiratory rehabilitation of coronavirus disease 2019 in adult]. Chin J Tuberc Respir Dis. 2020; 43: 308-314. doi:10.3760/CMA.J.CN112147-20200228-00206.

8. Lin $\mathrm{WL}$, Hon KL, Leung KKY, Lin ZX. Roles and challenges of traditional Chinese medicine in COVID-19 in Hong Kong. Hong Kong Med J. 2020; 26: 268-269.

9. Zhao H, Wu XD, Wang XX, Song JL, Pan L, Shen JG, et al. Wang Qingguo's cases and thoughts of differentiating and treating novel coronavirus pneumonia Zhao. Mod Chin Clin Med. 2020; 4: 17.

10. Wu Yong. Summary of experience and Xiang Xia Hospital Model China Daily [Internet]. China Daily; $2020 . \quad$ Available from: https://cn.chinadaily.com.cn/a/202003/13/WS5e6b8aa8a3107bb6b57a6628.html?from=grou pmessage\%20HYPERLINK\%20\%22https://cn.chinadaily.com.cn/a/202003/13/WS5e6b8aa8a31 07bb6b57a6628.html?from=groupmessage\&isappinstalled=0\%22\&\%20HYPERLINK\%20\%22htt ps://cn.chinadaily.com.cn/a/202003/13/WS5e6b8aa8a3107bb6b57a6628.html?from=groupm essage\&isappinstalled $=0 \% 22$ isappinstalled $=0$.

11. Yang Y, Islam MS, Wang J, Li Y, Chen X. Traditional Chinese medicine in the treatment of patients infected with 2019-new coronavirus (SARS-CoV-2): A review and perspective. Int J Biol Sci. 2020; 16: 1708-1717.

12. Zhao J, Tian SS, Yang J, Liu J, Zhang WD. Investigating the mechanism of Qing-Fei-Pai-Du-Tang for the treatment of novel coronavirus pneumonia by network pharmacology. Chin Tradit Herb Drugs. 2020; 4: 829-835.

13. The Centre for Health Protection (CHP) of the Department of Health. Latest situation of cases of COVID-19 (as of 20 May 2021). 2021. Available from: https://www.chp.gov.hk/files/pdf/local situation covid19 en 20210520.pdf.

14. 7th clinical practice guidelines on Covid-19 (implementation version) [Internet]. The National Health Commission of the People's Republic of China. 2020. Available from: http://www.gov.cn/zhengce/zhengceku/2020-03/04/content 5486705.htm.

15. 2nd clinical practice guidelines on COVID-19 with Chinese medicine (implementation version) [Internet]. Traditional Chinese Medicine Bureau of Guangdong Province. 2020. Available from: http://szyyj.gd.gov.cn/zwgk/gsgg/content/post 2902010.html.

16. Zhang Boli: The efficacy of TCM in the fight against COVID-19. Sci Technol Daily. 2020. Available from: http://www.china.org.cn/china/2020-04/06/content 75899176.htm.

17. Zheng XY. The guidelines of new drug discovery of Chinese medicine. Beijing: China Medicine Science and Technology Press; 2002.

18. Huang C, Zhu YB, Liu ZJ. Approaches for developing a system model for health evaluation based on body constitutions of traditional Chinese medicine and order parameters. J Chin Integr Med. 2012; 10: 375-379.

19. Wang J, Li YS, Ni C, Zhang HM, Li LR, Wang Q. Cognition research and constitutional classification in Chinese medicine. Am J Chinese Med. 2011; 39: 651-660.

20. China Association of Chinese Medicine. The classification and standard of body constitution in Chinese Medicine. World J Integr Trad Western Med. 2009; 4: 303-304. 
21. Hanania NA, Mannino DM, Yawn BP, Mapel DW, Martinez FJ, Donohue JF, et al. Predicting risk of airflo obstruction in primary care: Validation of the lung function questionnaire (LFQ). Respir Med. 2010; 104: 1160-1170.

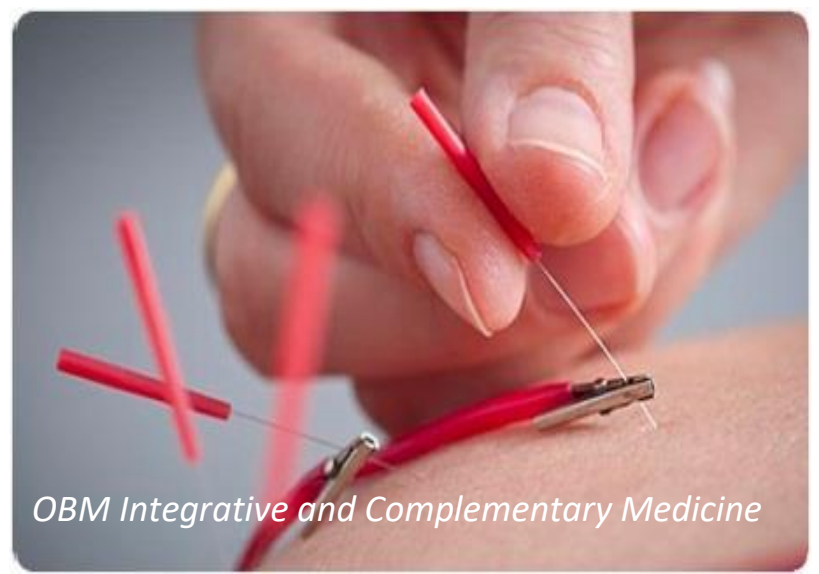

Enjoy OBM Integrative and Complementary Medicine by:

1. Submitting a manuscript

2. Joining in volunteer reviewer bank

3. Joining Editorial Board

4. Guest editing a special issue

For more details, please visit:

http://www.lidsen.com/journals/icm 